Dresden

\title{
ZU EINIGEN ASPEKTEN EINER TEXTSORTENBESCHREIBUNG MIT BEZUG ZUM BEGRIFF 'PROTOTYP'
}

Anlaß für die folgenden Überlegungen ist die Suche nach einem theoretischen Zugang zur Beschreibung und Erklärung des Einflusses situativer Elemente auf die sprachliche Ausprägung von Merkmalen von Textsorten.

Wenn Textexemplare bestimmten Textsorten zugeordnet werden sollen, ergeben sich bei einer Anzahl von Texten mehrere Möglichkeiten der Zuordnung. Wissen um diese Varianten in der Zuordnung muß z.B. Sprachlehrern vermittelt werden, damit sie in der Lage sind, Merkmale von häufig vorkommenden Textsorten zu erfassen und lehrbar zu machen. Außerdem erwerben Lerner Wissen über Merkmale von Textsorten, indem sie Erfahrungen bei der Lösung ausgewählter kommunikativer Aufgaben sammeln. Die Ergebnisse der Textkonstitution werden z.B. vom Lehrer eingeschätzt. Der Maßstab für diese Einschätzung ist noch zu wenig davon geprägt, daß sprachliche Realisierungen auch Textsortenmerkmale verdeutlichen und nicht nur relativ festgelegte Schritte in der Lösung kommunikativer Aufgabenstellungen.

Bisherige Ansätze der Textklassifikation eignen sich noch nicht in ausreichendem Maße dafür, daß Merkmalsbeschreibungen einer Textsorte für die Bewertung von sog. Ausdrucksleistungen im Schulunterricht herangezogen werden können. Sie lassen weiter die Frage offen, warum einzelne Textexemplare mehreren Textsorten zugeordnet werden können. Ein Grund für diese Probleme kann möglicherweise in der Auswahl der Klassifizierungskriterien für Texttypologien gesehen werden. W. Heinemann (1988, S. 14) meint, daß "Klassifizierungsansätze zu kurz (greifen), die ausschließlich textexterne Gegebenheiten (etwa die Ziele der Textproduzenten) als Klassifizierungskriterium ansetzen. Sie gehen im Grunde an der eigentlichen Textspezifik vorbei, da man dasselbe Anliegen u.U. mit Hilfe ganz unterschiedlich strukturierter Textexemplare erreichen kann. Umgekehrt erweist sich auch eine Typisierung, die sich ausschließlich auf sprachliche Daten stützt, als nicht zureichend, da nichtsprachliche Komponenten (beispielsweise situative oder intentionale Spezifika) keineswegs immer unmittelbar aus der jeweiligen Textstruktur abgeleitet werden können.

Daher gehen wir von der Annahme aus, daß das Textklassenwissen durch multidimensionale Zuordnung von prototypischen Repräsentationen auf unterschiedlichen Ebenen konstituiert wird. Danach können Texte unter unterschiedlichen Aspekten 
typisiert werden: auf einer funktionalen und einer situativen Ebene, unter generellen und speziellen Verfahrensaspekten, aus der Sicht der Textstruktur i.e.S. und - nicht zuletzt - ausgehend von unterschiedlichen Formulierungsmustern."

Dieser Ansatz der mehrdimensionalen Textklassenbeschreibung ließe neben der Zuordnung von Textexemplaren zu verschiedenen Textsorten auch zu, daß die unterschiedliche Ausprägung der Merkmale einer Textsorte in konkreten Texten in Abhängigkeit von situativen Gegebenheiten beschreibbar wird. Dabei kann berücksichtigt werden, daß Quantität und Qualität von Textsortenmerkmalen durch außersprachliche Bedingungen beeinflußt werden.

Sprachliche Realisierungen von Textsortenmerkmalen in konkreten Texten unterscheiden sich, weil einerseits die Sprecher oder Schreiber von einer unterschiedlichen Zuordnung der Texte zu Textsorten ausgehen und somit unterschiedliches Textklassenwissen aktivieren, das dann in den Texten zum Ausdruck gebracht wird. Ein Textexemplar wird von verschiedenen Individuen nicht nur anders verstanden und unterschiedlichen Textsorten zugeordnet, sondern umgekehrt entscheiden diese Individuen von ihrem Wissen ausgehend unterschiedlich bei der eigenen sprachlichen Äußerung, je nachdem welche Merkmale sie der Textsorte zuordnen, welche Merkmale sie als unbedingt zu realisieren ansehen und welche für sie von untergeordneter Bedeutung sind.

So zeigen sich beispielsweise Unterschiede in Schüleraufsätzen in der Weise, daß, obwohl die gleiche kommunikative Aufgabe zu lösen ist, zum einen eine Vorgangsbeschreibung deutlich wird, des weiteren auch Berichte über den vorgeführten Vorgang entstehen und sogar einige Texte Merkmale von Kommentaren aufweisen. Unterschiede in den Texten kommen teilweise dadurch zustande, daß die Schreiber unterschiedliches Textsortenwissen besitzen und aktivieren können (vgl. Janz, 1987). Wenn sich der Sprachunterricht unter anderem das Ziel stellt, die Lernenden zur aktiven und angemessenen Kommunikation zu befähigen, so kann über die Textsorte die Vermittlung zwischen Parametern der kommunikativen Situation und der entsprechenden Verwendung sprachlicher Mittel zur Realisierung von Textsortenmerkmalen erfolgen.

Eine Grenze von Erklärungsansätzen für das Entstehen von Realisierungsvarianten von Textsortenmerkmalen besteht z.B. darin, daß von vornherein von normgerechten und abgestuft defizitären Realisierungen eines Handlungstyps (wie beim Beschreiben von Vorgängen) ausgegangen wird. Damit bleibt offen, in welcher Art und Weise Normen im Prozeß der Textkonstitution im Hinblick auf das Entstehen von Varianten wirken. Offen bleibt ebenfalls die Frage, inwiefern in der konkreten Kommunikationssituation Normabweichungen entstehen, die sofort auf die Textkonstitution Einfluß haben können, die evtl. zu variablen Merkmalen der Textsorte werden können.

Nach Brinker (1985, S. 124) sind Textsorten "konventionell geltende Muster für komplexe sprachliche Handlungen und lassen sich als jeweils typische Verbindungen von kontextuellen (situativen), kommunikativ-funktionalen und strukturellen (grammatischen und thematischen) Merkmalen beschreiben. Sie haben sich in der Sprachge- 
meinschaft historisch entwickelt und gehören zum Alltagswissen der Sprachteilhaber; sie besitzen zwar eine normierende Wirkung, erleichtern aber zugleich den kommunikativen Umgang, indem sie den Kommunizierenden mehr oder weniger feste Orientierungen für die Produktion und Rezeption von Texten geben." Textsortenwissen gehört nach dieser Definition zum Alltagswissen. Der Muttersprachler bildet dieses Wissen vor allem für solche Textsorten aus, die im individuellen und Sozialisierungsalltag eine Rolle spielen. Dieses Wissen wird in der Regel empirisch erworben und kann eigentlich nicht expliziert werden. Im Sprachunterricht soll aber darüber hinaus Textsortenwissen erworben werden für Textsorten, die in vielfältigen Kommunikationsbereichen Bedeutung besitzen, und die Lernenden sollen befähigt werden, wenigstens Teile des Wissens explizieren zu können. Dadurch können Kriterien für die Einschätzung der Angemessenheit in der Lösung von kommunikativen Aufgaben abgeleitet werden, so daß z.B. bewertet werden kann, ob wesentliche Merkmale der Textsorte zum Ausdruck kommen und welche Varianten in der Realisierung der Merkmale noch angemessen erscheinen.

Um die kurz charakterisierten Zwecke zu erfüllen, ist eine Textsortenbeschreibung notwendig, die sowohl wesentliche Merkmale von Textsorten erfaßt, als auch die variable Realisierung der Merkmale in Texten in Abhängigkeit von z.B. situativen Bedingungen erklärbar macht. Vorteile einer solchen Beschreibung wären darin zu sehen, daß Lernenden nicht nur wesentliche Merkmale einer Textsorte bewußt gemacht werden könnten, sondern auch Varianten in der Realisierung der Merkmale, die durch konkrete Kommunikationssituationen hervorgerufen werden können.

Ein Ansatz zur Beschreibung von Textsorten über den Begriff Prototyp soll hier theoretisch diskutiert werden, ohne daß sich schon Möglichkeiten für empirische Überprüfungen andeuten lassen. Um den Einfluß situativer Elemente auf die Ausprägung der Textsorte zu erfassen und Beziehungen zwischen Situativem und sprachlichen Realisierungsweisen aufzudecken, ist der angenommene Zusammenhang von Textsorte und zugehörigen Textexemplaren ein Ausgangspunkt für die Beschreibung.

Wir nutzen folgende Problematisierung für einen Beschreibungsansatz: Harnisch u. Friese (1986, S. 52) gehen davon aus, daß "mit der Abbildung von Textsorten und ihren Sprach"mustern" in variabel ausfüllbaren Modellen allerdings das Problem (zusammenhängt), daß sich diese Modelle nur auf Grundmerkmale der thematischen, handlungstypischen und stilistisch-formulativen Seite der Texte beziehen und außerdem nur eine geringe Garantie dafür geben, daß im Modell der jeweiligen Textsorte die Menge aller zur Textsorte gehörenden Textexemplare erfaßt sind. Wirklich allgemeingültige Muster beispielsweise von Appellen, Festreden ... usw. führen meist zu äußerst merkmalsarmen Modellen, die auch nur von geringer Effizienz für die Handlungsanleitung sind. Viel wirksamer und für die ontogenetische Ausbildung von Textnormen viel entscheidender sind innerhalb eines auf konstanten, allgemeinen Merkmalen beruhenden Textmodells typische, exemplarische Ausprägungen, die eine Textsorte und ihre sprachliche Ausprägung zwar nicht als logische Klassenbildung auf der Basis invarianter Merkmale, sondern als Prototyp auf der Basis dominanter Merk- 
male widerspiegeln." Textsorte wird hier als Kategorie verstanden, wobei sie aus dominanten Merkmalen gebildet wird. Die dominanten Merkmale finden sich im sog. besten Exemplar der Klasse von Textexemplaren, die die Textsorte als Modell oder Muster konstituieren. Der Begriff 'Prototyp' wird als bestes Exemplar bzw. Beispiel, bester Vertreter oder zentrales Element einer Kategorie eingeführt. Weiter heißt es bei Kleiber (1993, S. 31): "Es handelt sich also um eine Fachbedeutung, die sich von der umgangssprachlichen Bedeutung "erstes, vor der Serienproduktion hergestelltes Exemplar eines Modells (einer Maschine oder eines Autos)" unterscheidet. Die grundlegende Idee besteht darin, daß sich die Kategorien nicht aus Exemplaren zusammensetzen, die im gleichen Verhältnis zur überdachenden Kategorie stehen, sondern daß es Exemplare gibt, die bessere Vertreter sind als andere. So ergaben die Antworten der von E. Rosch (1973) befragten Personen, daß der Apfel das beste Exemplar für die Kategorie Obst ... darstelle, während Olive am wenigsten repräsentativ sei; dazwischen findet man (in der Reihenfolge absteigender Repräsentativität) Pflaume, Ananas, Erdbeere und Feige.

Wie dieses Beispiel zeigt, hängt der Begriff des Prototyps ursprünglich in höchst bedeutsamer Weise mit den Individuen zusammen: Der Prototyp ist das Exemplar, das von den Sprechern als bestes anerkannt wird." Es muß hier die Frage aufgeworfen werden, inwiefern der Grad der "Vertrautheit" mit den Exemplaren und inwiefern die Vorstellungen über sie nicht eine große Rolle spielen, wenn ein Exemplar als Prototyp benannt wird.

Wenn Textsorten als Muster für komplexe sprachliche Handlungen betrachtet werden, die sich in der Sprachgemeinschaft historisch entwickelt haben und zum Alltagswissen der Sprachteilhaber gehören (s.o.), dann besteht ihr Wesen darin, daß sie Klassen von Objekten bilden, die invariante Merkmale besitzen. Diese Merkmale lassen die Zuordnung von jedem Textexemplar zu einer Textsorte zu, sagen aber nichts aus über die unterschiedliche Qualität der Merkmale und über die unterschiedliche Ausprägung der Merkmale in Textexemplaren in Abhängigkeit von nichtsprachlichen Bedingungen in der Kommunikation. Wenn wir invariante Merkmale von Textsorten annehmen, die eine Abstraktion über einer relativ homogenen Menge von Textexemplaren zulassen, und bei der Beschreibung auch davon ausgehen, daß es Merkmale von Textsorten gibt, die unterschiedlich in Texten ausgeprägt sind und die unter Umständen durch bestimmte situative Bedingungen erst konstituiert werden, dann müßten auch diese Merkmale $\mathrm{zu}$ erfassen sein.

Die konkreten Ausprägungen einer Textsorte, die in ihrer Gesamtheit nicht erfaßt werden können, weisen in der sprachlichen Realisierung von Textsortenmerkmalen diese dominanten Merkmale in unterschiedlichen Ausprägungsgraden auf. Wenn wir die theoretischen Überlegungen zum Begriff des Prototyps in der Logik nutzen, die in der linguistischen Semantikforschung (Blutner 1985), (Kleiber 1993), (Meinhard 1984) und in der kognitiven Psychologie (Klix 1976, S. 169 f.) modifiziert werden, ergibt sich eine mögliche Erklärung für das Auftreten von Ausprägungsgraden oder von Varianten einer Textsorte. Möglicherweise ist auch eine Erklärung für die unterschiedliche Zuord- 
nung von Textexemplaren zu mehreren Textsorten zu finden. Meinhard (S. 60) stellt fest, daß sich "durch die Einführung des Begriffs der varianten Merkmale, der neben den üblichen der invarianten Merkmale der Wortbedeutung tritt, ... prototypische Merkmale als ausgezeichnete Teilmenge der invarianten und/oder varianten Merkmale darstellen" lassen. Wichtigstes Element der Definition von Prototypen, das genutzt werden könnte, ist: "Prototypische Merkmalsstrukturen erfassen die Eigenschaften, die einem besonders typischen Vertreter einer Objektklasse zukommen, somit auch Eigenschaften, die nicht allen Mitgliedern der Objektklasse zukommen müssen, die also nicht invariant sind" (Meinhard, S. 61). Auch Klix (1976, S. 170) hebt diese möglichen Eigenschaften eines Prototyps hervor, wenn er feststellt: "Es kann nicht ausgeschlossen werden, daß einer derartigen Prototypbildung auch eine bildlich-anschauliche Repräsentation entsprechen kann ... Etwas grundsätzlich Neues ... besteht darin, daß die relevanten Merkmale einer Klasse nicht notwendig bei jedem Exemplar der Klasse vertreten sein müssen. Mit anderen Worten: Die Merkmale und ihre Ausprägungsgrade sind bezüglich des Zugehörigkeitsgrades - untereinander kompensierbar."

Diese Bestimmung von Prototypen ließe sich auf eine Textsortenbeschreibung wie folgt übertragen: Eine Textsorte weist invariante Merkmale auf, die sich aus der Zuordnung zu dem oder den in der Textsorte dominierenden Handlungstypen ergeben und die jeweils typische Verbindungen von kontextuellen, kommunikativ-funktionalen und strukturellen Textmerkmalen reflektieren. Merkmale der Textsorte, die nicht in jedem Textexemplar realisiert werden und/oder in unterschiedlicher qualitativer Ausprägung in den Textexemplaren auftreten, sind dann aus Differenzierungen zwischen den verschiedenen Individuen und aus verschiedenartigen situativen Gegebenheiten bei der Lösung von kommunikativen Aufgaben ableitbar. Auf diese Weise wäre stärker die Veränderlichkeit der Textsorten zu kennzeichnen. Wir würden dann evtl. der Beschreibung von Textsorten nach D. Neuendorff (1988, S. 544) nahe kommen. Sie sagt: "Textsorten sind zu verstehen als historisch und kulturell sich verändernde Verständigungskonzepte einer Gesellschaft, die mit mehr oder minder stark festgelegten Funktionen verbunden sind. Sie werden realisiert durch Textsortengroßstrukturen, welche die Verlaufsstruktur einer Textsorte wiedergeben. Diese wiederum sind geprägt durch das Wissen der Kommunizierenden über Handlungsmuster und deren Sequenzierung. Indem diese aber als auf mindestens einer (mit der konventionellen Funktion der Textsorte in Beziehung stehenden) dominierenden illokutiven Handlung und den sie stützenden illokutiven Handlungen basierend interpretiert werden, die sich wiederum im konkreten Textexemplar einer Textsorte realisieren, stellt sich die Beziehung zum konkreten Text her."

Ausgehend von diesen Überlegungen zur Standardversion des Begriffs Prototyp in der Semantiktheorie könnten neue Erklärungsrichtungen in der sog. erweiterten Version der Prototypensemantik (Kleiber, S. 109 ff.; vor allem S. 114 ff.) Beachtung in der Textlinguistik finden. 


\section{LITERATUR}

Blutner, R.: Prototyptheorien und strukturelle Prinzipien der mentalen Kategorisierung. In: LS/ZISW/A, H. 125. Berlin 1985.

Brinker, K.: Linguistische Textanalyse. Berlin 1985.

Harnisch, H.; Friese, E. (Mitarbeit): Funktional relevante Faktoren bei der variablen Verwendung von Lexik und Grammatik in Handlungstypen und Textsorten. In: Potsd. Forschungen, Reihe A, H. 82, Potsdam 1986.

Heinemann, W.: Zur Rolle des Stils bei einer Mehrebenenklassifikation von Texten. In: Textlinguistik, Heft 14, Dresden 1988.

Janz, Chr.: Untersuchungen zur Ausprägung des Kommunikationsverfahrens Beschreiben in Klasse 5 und 9 in Abhängigkeit von Elementen der konkreten sozialen Situation. Ein Beitrag zur Erfassung soziolinguistischer Aspekte in der funktional-kommunikativen Sprachbeschreibung. Diss. (A), Dresden 1987.

Kleiber, G.: Prototypensemantik. Eine Einführung. Tübingen 1993.

Klix, F.: Psychologische Beiträge zur Analyse kognitiver Prozesse. Berlin 1976.

Meinhard, H.-J.: Invariante, variante und prototypische Merkmale der Wortbedeutung.

In: Zs. für Germanistik 5 (1984) 1.

Neuendorff, D.: Textsorte als Handlung - $\mathrm{Zu}$ einigen Aspekten einer prozeduralen Textsortenbeschreibung. In: Neuphilologische Mitteilungen, 4 LXXXIX 1988, Helsinki.

\section{Zusammenfassung}

Theoretische Überlegungen zum Begriff 'Prototyp' in der Logik, in der linguistischen Semantikforschung und in der kognitiven Psychologie werden in bezug auf die Bestimmung von Textsorte als Kategorie diskutiert. Die sog. Standardversion in der Prototypensemantik bietet Ansatzpunkte für eine Textsortenbeschreibung unter prozeduralem Aspekt. Diese und die sog. erweiterte Version können Erklärungsrichtungen zu folgenden Problemen weisen: Warum können einzelne Textexemplare mehreren Textsorten zugeordnet werden? Wie lassen sich Differenzierungen in der Ausprägung von Textsortenmerkmalen in konkreten Textexemplaren oder das Nichtausgeprägtsein von Merkmalen erklären?

Povzetek

NEKATERI VIDIKI OPISA BESEDILNIH VRST Z OZIROM NA POJEM "PROTOTIP"

Teoretični pojem "prototip" je obravnavan v logiki, v raziskavah na področju jezikovne semantike in $v$ kognitivni psihologiji v okviru besedilne vrste kot kategorije.

Tako imenovana standardna inačica prototipične semantike služi kot izhodišče za opis besedilnih vrst $s$ proceduralnega vidika. Ta in t.i. razširjena različica sta možni iztočnici za pojasnitev naslednjih vprašanj: zakaj lahko uvrstimo nekatera besedila $v$ različne besedilne vrste, zakaj se značilnosti določene besedilne vrste $v$ nekaterih konkretnih besedilih pogosteje pojavljajo kot $\mathrm{v}$ drugih in kako pojasniti dejstvo, da v določenih besedilih tovrstnih značilnosti ni? 